\title{
USO DO PROTOCOLO DE SAÚDE DA MULHER NA PREVENÇÃO DO CÂNCER DE COLO DO ÚTERO
}

\author{
THE USE OF WOMEN'S HEALTH RECORD \\ IN CERVICAL CANCER PREVENTION
}

\section{EL USO DEL PROTOCOLO DE SALUD DE LA MUJER EN LA PREVENCIÓN DEL CÁNCER CERVICAL}

\author{
Joyce Carolyne Ribeiro de Holanda ${ }^{1}$ \\ Maria Helloysa Herculano Pereira de Oliveira de Araújo \\ Wezila Gonçalves do Nascimento ${ }^{3}$ \\ Maeli Priscila Alves Gama ${ }^{4}$ \\ Claudia Santos Martiniano Sousa ${ }^{5}$
}

Como citar este artigo: Holanda JCR, Araújo MHHPO, Nascimento WG, Gama MPA, Sousa CSM. Uso do protocolo de saúde da mulher na prevenção do câncer de colo do útero. Rev baiana enferm. 2021;35:e39014.

Objetivo: analisar o uso do protocolo de saúde da mulher na prevenção do câncer de colo do útero por enfermeiros na Atenção Básica. Método: estudo de caso, exploratório, de abordagem qualitativa. Os participantes foram enfermeiros da Estratégia Saúde da Família. Utilizou-se como técnica de coleta de dados a entrevista semiestruturada, analisada pela Análise de Conteúdo do tipo categorial temática. Resultados: o enfermeiro realiza o acolhimento limitado à queixa da mulher motivada por demanda espontânea e apresenta autonomia para a realização do citopatológico, embora nem todos realizem avaliação do resultado desse exame. Conclusão: a análise do uso do protocolo de saúde da mulher permitiu constatar-se uma discrepância entre as ações realizadas por enfermeiros na Atenção Básica, que ora estavam de acordo com o Protocolo de Atenção Básica, ora divergiam de suas normativas.

Descritores: Atenção Primária à Saúde. Saúde da Mulher. Câncer de Colo do Útero. Prevenção Primária. Protocolos Clínicos.

Objective: analyzing the use of the women's health record in the prevention of cervical cancer by nurses in Primary Care. Method: this is an exploratory case study of a qualitative approach. The participants were nurses of the Family Health Strategy. The data collection technique used was the semi-structured interview, analyzed by Content Analysis of the thematic categorical type. Results: the nurse performs the reception limited to the complaint of women motivated by spontaneous demand and has autonomy to perform the cytopathological examination, although not all perform the evaluation of the result of this examination. Conclusion: the analysis of the use of the women's health record allowed a discrepancy to be found between the actions performed by nurses in Primary Care, which were sometimes in accordance with the Primary Care Record, sometimes diverging from its norms.

Descriptors: Primary Health Care. Women's Health. Cervical Cancer. Primary Prevention. Clinical Records.

\footnotetext{
Enfermeira. Pesquisadora Independente. Campina Grande, Paraíba, Brasil. http://orcid.org/0000-0002-1473-7324.

Enfermeira. Universidade Federal da Paraíba. Enfermeira da Prefeitura Municipal de Recife (PE) e da Policlínica e Maternidade Professor Arnaldo Marques. Campina Grande, Paraíba, Brasil. mh.herculano@gmail.com. http://orcid.org/0000-0003-3002-4639.

3 Enfermeira. Mestre em Saúde Pública. Universidade Estadual da Paraíba. Campina Grande, Paraíba, Brasil. http://orcid.org/0000-0002-9295-1385.

4 Enfermeira. Pesquisadora Independente. Campina Grande, Paraíba, Brasil. http://orcid.org/0000-000 I-6787-7745.

Enfermeira. Doutora em Ciências da Saúde. Professora da Universidade Estadual da Paraíba. Campina Grande, Paraíba, Brasil. http://orcid.org/0000$0001-6662-6610$
} 
Objetivo: analizar el uso del protocolo de salud de la mujer en la prevención del cáncer cervical por parte de las enfermeras de Atención Primaria. Método: se trata de un estudio de caso exploratorio con enfoque cualitativo. Los participantes fueron enfermeras de la Estrategia de Salud de la Familia. La técnica de recogida de datos utilizada fue la entrevista semiestructurada, analizada mediante Análisis de Contenido de tipo categórico temático. Resultados: el enfermero realiza el acolchado limitado a la piel de la mujer motivado por la demanda espontánea y se muestra autónomo para la realización del citopatológico, aunque no todos realizan la evaluación del resultado de este examen. Conclusión: el análisis del uso del protocolo de salud de la mujer permitió constatar una discrepancia entre las acciones realizadas por los enfermeros en la Atención Básica, que a veces estaban de acuerdo con el Protocolo de Atención Básica, y a veces divergían de sus normativas.

Descriptores: Atención Primaria de Salud. Salud de la Mujer. Cáncer de Cuello de Útero. Prevención Primaria. Protocolos Clínicos.

\section{Introdução}

O câncer do colo de útero é o segundo tipo mais incidente entre as mulheres brasileiras, porém é de fácil detecção por meio da coleta de material para o exame preventivo citopatológico, prioritariamente entre as idades de 25 a 59 anos e com vida sexual ativa. O intervalo entre os exames deve ser de três anos, após resultado negativo em dois exames anuais consecutivos ${ }^{(1)}$.

No Brasil, em 2020, foram estimados 16.710 novos casos de câncer do colo do útero, com um risco aproximado de 15,38 casos a cada 100 mil mulheres. No ano de 2019, essa neoplasia foi responsável por 6.596 óbitos, representando uma taxa ajustada de mortalidade de 5.33/100 mil mulheres, conforme dados disponibilizados pelo Instituto Nacional de Câncer ${ }^{(2)}$.

O principal fator de risco associado ao alto índice de desenvolvimento do câncer de colo do útero é a infecção pelo Papilomavírus Humano (HPV), especialmente os subtipos 16 e 18, responsáveis por $70 \%$ dos cânceres cervicais. Além disso, fatores como atividade sexual desprotegida, imunossupressão, tabagismo, multiparidade, dieta inadequada, uso de pílulas anticoncepcionais orais por um longo período de tempo (mais de cinco anos), múltiplos parceiros sexuais, início precoce da atividade sexual e idade acima de 30 anos, podem influenciar no desenvolvimento da infecção pelo HPV e progressão para lesões precursoras ou câncer ${ }^{(3)}$.

A prevenção primária do câncer de colo do útero inicia-se com a oferta de vacinação contra o HPV, orientações sobre o uso de preservativo e o combate ao tabagismo. Essas condutas não reduzem a necessidade do rastreamento por meio do exame citopatológico, com foco na detecção precoce de lesões pré-cancerosas. Assim, é importante realizar busca ativa de mulheres dentro da população-alvo e com exames em atraso, dar seguimento aos casos com resultados alterados (encaminhar para serviços de referência, médico especialista ou realização de exame mais complexo) e educação em saúde ${ }^{(3)}$.

Para a operacionalização dessas ações, o Ministério da Saúde vem produzindo materiais técnicos que visam orientar o processo de trabalho na Atenção Primária à Saúde. Esses materiais são os Cadernos de Atenção Básica, que foram lançados em 2006 e 2013. O mais recente material com o objetivo de potencializar a atuação das equipes de saúde é o documento "Protocolos da Atenção Básica: Saúde das Mulheres”, publicado em 2016 ${ }^{(4)}$. Esta publicação possui abrangência nacional e contempla as seguintes linhas orientadoras: atenção aos problemas/queixas mais comuns em saúde das mulheres, atenção às mulheres no pré-natal de baixo risco, puerpério e promoção do aleitamento materno, planejamento reprodutivo, prevenção de câncer de colo do útero, prevenção do câncer de mama, atenção às mulheres no climatério e atenção às mulheres em situação de violência sexual e/ou doméstica/intrafamiliar ${ }^{(4)}$.

No contexto da prevenção do câncer de colo do útero, o enfermeiro desempenha diversas atividades, sendo responsável pela realização da 
consulta de enfermagem e coleta de material para o exame citopatológico. Além disso, orienta sobre medidas preventivas, gerencia o provimento de recursos materiais, encaminha resultados suspeitos e pratica diversas ações educativas junto à equipe de saúde e à comunidade ${ }^{(5)}$.

Torna-se perceptível que a atividade que o enfermeiro exerce na Estratégia Saúde da Família (ESF) é de total importância não só para o desenvolvimento de práticas embasadas, inclusive em protocolos, que podem ser definidos para todas as atividades de enfermagem em todos os níveis de atenção ${ }^{(6)}$, como também para o desempenho da equipe na qual está inserido nos serviços de saúde.

Com base no exposto, esta pesquisa teve como perguntas norteadoras: Os enfermeiros da Estratégia de Saúde da Família (ESF) do município de Campina Grande (PB) conhecem e utilizam o Protocolo da Atenção Básica para nortear suas ações de saúde da mulher no que diz respeito à prevenção do câncer de colo do útero? Quais as possíveis facilidades e dificuldades no uso de protocolos no processo de trabalho do enfermeiro?

Este artigo tem como objetivo analisar o uso do protocolo de saúde da mulher na prevenção do câncer de colo do útero por enfermeiros na Atenção Básica.

\section{Método}

Trata-se de um estudo de caso, exploratório, de abordagem qualitativa, guiado pela ferramenta COREQ. O cenário da pesquisa foi a ESF do município de Campina Grande (PB).

Participaram do estudo enfermeiros que atenderam aos seguintes critérios de inclusão: atuar na ESF do município supracitado, visto que realizam o rastreamento do câncer de colo do útero. Como critério de exclusão, foi considerado apenas não estar exercendo a função assistencial nas equipes Saúde da Família.

Para a amostra, foram sorteados aleatoriamente 5 enfermeiros de cada distrito sanitário, totalizando 40 participantes. Durante a coleta de dados, houve perda de um participante, por não haver disponibilidade para a participação na pesquisa. Desse modo, o novo total incluiu 39 enfermeiros.

Os dados foram coletados entre os meses de maio e junho de 2017, no local onde o enfermeiro trabalhava, em horário que ele julgou possível. Além da entrevista com questões semiestruturadas, utilizou-se um instrumento de coleta de dados constituído de duas partes: a primeira, com questões referentes à caracterização dos sujeitos; e a segunda, com perguntas relativas ao objetivo da pesquisa. A construção do instrumento de coleta de dados fundamentou-se no Protocolo da Atenção Básica - Saúde das Mulheres de 2016, publicado pelo Ministério da Saúde ${ }^{(4)}$.

Para a validação do instrumento de pesquisa, foi realizado teste piloto com um enfermeiro, visando avaliar os possíveis vieses de interpretação durante a realização da pergunta, bem como identificar se as questões formuladas atendiam ao objetivo da pesquisa.

As entrevistas foram gravadas em gravador digital em dupla entrada. Os entrevistados assinaram o Termo de Autorização para gravação de voz. A gravação das entrevistas foi transcrita na íntegra e constituiu o corpus que foi submetido à análise. Para garantir o anonimato, os participantes foram identificados pela sigla ENF seguida do número de ordem das entrevistas.

A análise de conteúdo foi do tipo categorial temática, que se organiza em três fases: pré-análise, com a leitura flutuante do material; exploração do material, que consiste na codificação e decomposição em função das categorias previamente elencadas; e tratamento dos resultados como unidades de análise, os fragmentos das falas dos sujeitos entrevistados com base em inferências e interpretações ${ }^{(7)}$.

O presente estudo faz parte da pesquisa intitulada "Conhecimento e a Utilização dos Protocolos de Saúde da Mulher por Enfermeiros da Atenção Primária à Saúde", na qual são analisados o conhecimento e a utilização dos protocolos por esses profissionais, em suas oito dimensões mencionadas. O protocolo de pesquisa foi aprovado pelo Comitê de Ética em 
Pesquisa da Universidade Estadual da Paraíba, sob Parecer $\mathrm{n}^{\circ}$ 1881700, Certificado de Apresentação de Apreciação Ética (CAAE) n. 602782163.0000 .5187 .

\section{Resultados}

A amostra da pesquisa constituiu-se de 39 enfermeiros da ESF distribuídos nos 8 distritos do município de Campina Grande (PB). A maioria era do sexo feminino $(94,9 \%)$, na faixa etária de 25 a 39 anos (46,2\%) e casados (74,0\%).

Com relação ao tempo de formação, 35,9\% dos enfermeiros entrevistados estavam formados há mais de uma década. No que diz respeito à realização de pós-graduação, mais de 92\% dos entrevistados possuíam o título de especialista. Destes, nove enfermeiros realizaram mais de duas especializações, sendo o maior percentil (63,6\%) constituído de especialistas em Saúde Pública/Saúde da Família/Coletiva Comunitária.

No quesito capacitação, 97,4\% dos entrevistados realizaram alguma capacitação em Saúde da Mulher e 92,3\% concluíram pelo menos duas capacitações. Assim, 76,9\% realizaram capacitação em Prevenção do Câncer de Colo do Útero, e 36,0\% a concluíram há menos de cinco anos.

\section{Acolbimento com escuta qualificada}

Entre os enfermeiros entrevistados, alguns informaram que realizavam acolhimento às mulheres que procuravam o serviço e tomavam por base suas queixas, como se observa nas falas a seguir:

Se ela tem queixa. Se tá fazendo pra rotina ou se ela tem alguma queixa, e aí ela vai vir com queixa de dor pélvica, dor pra urinar, corrimento, sangramento. E aí, dependendo da queixa dela, dou prosseguimento [...] (Enf. 7).

Procuro ver o motivo que ela veio para aquela consulta, qual a queixa dela no momento. (Enf. 15).

\section{Avaliação global da mulher}

No atendimento das mulheres que irão submeter-se ao exame citológico, os enfermeiros entrevistados referiram realizar abordagem integral, como revela a fala a seguir:

\begin{abstract}
Antes, a gente faz uma consulta integral. Então, eu vou ver o estado geral de saúde da mulher, vou fazer as perguntas que são pertinentes, se ela tem alguma queixa e, das queixas, eu vou ver se estão relacionadas ao aparelho geniturinário, na parte ginecológica dela. Tudo a gente vai ver ali. E vai fazer o preenchimento daquela ficha que tem a história todinha. (Enf. 28).
\end{abstract}

No presente estudo, outros enfermeiros referiram limitar-se à queixa atual e à realização da coleta do material para exame citológico:

Olha... Eu não gosto de entrar na intimidade dela e perguntar com quantas pessoas já teve relação nem nada disso. Então, procuro avaliar qual a queixa dela, o valor da sua pressão arterial. E quando faz muito tempo que ela fez exames de rotina, aproveito a oportunidade para solicitar. (Enf. 1).

Afirmaram realizar abordagem sindrômica com as usuárias nas quais foi identificada alguma alteração no momento da coleta do citológico, como visto a seguir:

Sim. Dependendo das alterações, né? Se for uma, algum tipo de leucorreia, algo que possa ser resolvido aqui mesmo, a gente vê. Se tiver medicação, a gente já trata. Dependendo da clínica, a gente já usa o protocolo [...] (Enf. 18).

Sim. Eu consulto o manual do COREN [Conselho Regional de Enfermagem] e prescrevo o medicamento, dependendo das queixas da paciente e dos achados na bora da coleta do citológico. (Enf. 3).

Dos enfermeiros que participaram do estudo, alguns referiram conhecer as mulheres que se encontravam em situações especiais para coleta do exame citológico, como mostram as falas seguintes:

As gestantes, as mulberes que estão no climatério ou menopausa e as que fizeram bisterectomia. (Enf. 1).

Sim. Sei que são as mulheres grávidas, com menopausa e as que fizeram a retirada do útero. Também tem as com HIV, porque tem que fazer o exame com mais frequência. (Enf. 5).

\section{Plano de cuidados na prevenção do câncer} de colo do útero

Os participantes afirmaram ter autonomia para realizar a consulta de retorno, como revelam as falas a seguir:

Geralmente, a consulta de retorno é feita com o médico ou então, é... Como a gente recebe o resultado previamente, a gente avalia. Se for um exame mais simples, um resultado mais simples, dentro do protocolo do Ministério, que a gente possa resolver, a enfermeira resolve também. (Enf. 18). 
Sim. Depois que elas fazem o citológico, eu peço pra elas, quando elas tiverem com o resultado agendar a consulta, pra passar por mim ou pelo médico. (Enf. 20).

A respeito do encaminhamento das usuárias para serviços de referência, alguns dos entrevistados disseram que realizavam o encaminhamento das usuárias para colposcopia após o resultado do citológico, como mostram as falas:

O encaminhamento para colposcopia só pode ser feito após a entrega do resultado do citológico. Como é a médica quem analisa os resultados, geralmente, é ela quem faz o encaminhamento. (Enf. 5).

Quando o resultado, o resultado que a gente recebe do laboratório, ele já sugere a colposcopia, entendeu? E quando ele não vem com essa sugestão, vejo o parâmetro que a gente segue né, e aí se for NIC II, a gente pode pedir a colposcopia. (Enf. 33).

Entretanto, outros enfermeiros, referiram realizar encaminhamento dessas usuárias para colposcopia mediante a identificação de alterações visíveis durante a coleta do citológico, como corroboram as falas:

Encaminho. Justamente quando ela tem uma lesão no colo do útero, que eu identifiquei na bora do citológico ou se ela vem com alguma outra alteração no exame de citológico dela, que sugestione a coleta da colposcopia, o exame mais detalhado. (Enf. 12).

[...] porém, quando a gente identifica uma situação, visivelmente, que chama a atenção, a gente pede pra fazer esta avaliação criteriosa, mesmo assim. (Enf. 14).

Quanto à realização de ações educativas com as usuárias, para estimulá-las a realizarem os exames preventivos, alguns enfermeiros referiram realizar essas ações na sala de espera, em grupos ou por meio de palestras, como esclarecem as falas:

Sim. Sempre que possível, a equipe realiza educação em saúde por meio da sala de espera. (Enf. 1).

Geralmente fazemos em conjunto com o pessoal do NASF [Núcleo Ampliado de Saúde da Família], com estudantes que vêm estagiar e com nossa unidade. Então, sempre tentamos realizar momentos na sala de espera ou convidamos as usuárias para uma palestra. (Enf. 5).

\section{Facilidades e/ou dificuldades encontradas na implementação de ações de prevenção em Câncer de Colo do Útero}

Entre as facilidades encontradas, identificou-se a busca espontânea pelo exame e o vínculo com o serviço, como se observa nas falas:
Bom. Aqui na minha unidade, especificamente, eu não tenho dificuldade. Eu tenho uma demanda assídua, que procura o serviço, independente da divulgação ou não. Ela procura o serviço [...] E tenho uma demanda fiel [...] (Enf. 26).

A facilidade é que elas têm um vínculo muito grande já. A maioria delas já tem um vínculo comigo, e aí muitas delas já se sentem à vontade por conta desse vínculo que já foi formado [...] elas já se sentem mais à vontade a realizar o exame. (Enf. 30).

A baixa adesão das usuárias ao exame e a falta de busca ativa foram ressaltadas por outros enfermeiros como dificuldades encontras nesse processo, como mostram as seguintes falas:

\begin{abstract}
A única dificuldade, que eu acho que é a milenar [risos] é a não adesão das mulheres. Muitas vezes, a gente pega uma mulher com mais de 10 anos, que não veio fazer o citológico [...] Assim... mas a maior dificuldade é isso... É a cultura mesmo, que elas ainda não [...] sentem muito ainda, o tabu, com relação ao citológico. (Enf. 15).
\end{abstract}

Ainda existe o medo de vir realizar o exame, da vergonha. Então, isso é uma coisa que ainda existem muitas pessoas que não procuram o serviço. Isso ainda é uma barreira pra gente. Como enfermeiro, procuro orientar essas pessoas e aí, geralmente, quando ela não quer fazer na unidade, porque está vendo aquele profissional, tento conversar e sensibilizar a mulher para tratar da saúde dela. (Enf. 24).

Dificuldade por falta de atualização, treinamento do profissional, também foi elencado como se observa na fala:

Eu acho que a gente deveria estar mais atualizada, porque, como eu estava dizendo, a gente não tem treinamento há muito tempo. Como a medicina todo dia cresce a ciência, a gente vai ficando para trás. Eu acho que deve ter coisa que a gente nem sabe e a gente deixa de atuar. (Enf. 11).

\section{Discussão}

Dentre os pontos de destaque no protocolo, a escuta qualificada é fundamental para a resolutividade do serviço, pois essa escuta facilita o atendimento e o encaminhamento das demandas apresentadas pelo usuário ${ }^{(8)}$.

Considera-se importante que o enfermeiro, ao abordar a mulher no citológico, aja de modo diferenciado, garantindo as condições necessárias para que ela possa conversar sobre a doença e conhecer como preveni-la. Deve ser ainda garantido o respeito à sua intimidade e à sua privacidade $^{(9)}$.

Ao tomar como referência de abordagem a perspectiva da enfermagem humanística, o 
enfermeiro propicia uma atenção voltada à promoção da saúde da mulher, com base no conhecimento sobre seu corpo e sua sexualidade, por meio de uma conversa aberta e empática ${ }^{(10)}$.

Todavia, a construção de vínculo entre o usuário e o profissional fica comprometida, quando a abordagem é limitada e implica em um atendimento rápido e mecânico, desconsiderando o processo relacional ${ }^{(11)}$, como ocorre nos casos relatados por alguns enfermeiros. Segundo estes, quando as usuárias procuram a unidade básica de saúde para a realização do citopatológico, são levadas em consideração apenas a queixa atual, coleta do material cervicovaginal e solicitação de exames de rotina. A não abordagem da intimidade das mulheres pelo enfermeiro reforça a busca pelo citopatológico com base em uma queixa dissociada do caráter preventivo do rastreamento. Nesse sentido, verifica-se a necessidade de ênfase na escuta e no diálogo, pois o vínculo afetivo é essencial para promoção da saúde sexual e reprodutiva ${ }^{(10)}$.

A saúde sexual e reprodutiva é considerada um dos eixos prioritários da atenção primária à saúde e também das diretrizes da Política Nacional de Assistência Integral à Saúde da Mulher. A constituição desse campo remete à autonomia, segurança e liberdade de tomar decisões sobre as formas de expressar e viver a sexualidade, sem risco de doenças e discriminação. Para tanto, deve-se garantir assistência clínica no pré-natal, parto e puerpério, realização de atividades educativas, atendimento às infecções sexualmente transmissíveis (IST) e outras afecções do aparelho reprodutor, encaminhamento e orientações quanto ao tratamento da infertilidade, cuidado nos casos de aborto previstos em lei, dentre outras ${ }^{(12)}$.

Abordar de maneira integral a saúde sexual e reprodutiva da mulher pode fomentar medidas preventivas para o câncer de colo do útero, por possibilitar a mitigação de alguns fatores de risco, como, por exemplo, atividade sexual desprotegida, múltiplos parceiros sexuais e infecção pelo HPV. Entretanto, o predomínio do enfoque da abordagem na sexualidade, na reprodução, no rastreamento do câncer de colo do útero, no planejamento reprodutivo seletivo para alguns contraceptivos, na restrita abordagem de vulnerabilidades, dentre outros, torna-se um desafio para a efetivação do cuidado prestado pelas equipes de saúde da família voltados à saúde sexual e reprodutiva ${ }^{(13)}$.

Segundo o protocolo, o enfermeiro pode realizar abordagem sindrômica - identificação de sinais e sintomas para seguimento de tratamento oportuno - durante a coleta do exame citopatológico, na presença de secreção vaginal anormal ou de colo friável e, independente desta abordagem, dar continuidade à rotina de rastreamento. Em caso de anormalidades, como sangramento fora do período menstrual ou lesões suspeitas, a usuária deve ser encaminhada para uma avaliação especializada. Em casos como, por exemplo, o cisto de Naboth, que não demanda intervenção, o enfermeiro deve apenas observar $^{(4)}$. Desse modo, considera-se relevante a abordagem sindrômica de atuação profissional, por possibilitar o tratamento precoce e evitar complicações provindas das IST, como forma de interceptar a transmissão ${ }^{(14)}$.

O protocolo ${ }^{(4: 178)}$ diz que necessitam de avaliação de serviços de referência ou de especialista e, portanto, devem ser encaminhados, os casos de:

\begin{abstract}
Lesão suspeita ao exame especular; Resultado de um colpocitológico com ASC-H; LIE ou SIL de alto grau ou carcinoma in situ; resultado de dois colpocitológicos consecutivos com ASC-US; LIE OU SIL de baixo grau; [...] Resultado de biópsia de colo com: neoplasia invasora (carcinoma epidermoide/adenocarcinoma); carcinoma microinvasor; displasia cervical grave, LIE alto grau (NIC 2/3); Resultado de colpocitologia com: células malignas ou carcinoma invasor; AGC (células glandulares atípicas de significado indeterminado).
\end{abstract}

Os enfermeiros, em seus discursos, evidenciaram a preocupação com o diagnóstico e o tratamento oportunos para as mulheres que apresentavam alteração no exame citológico. A preocupação demonstrada é coerente com pesquisas realizadas acerca da importância dessas intervenções. A redução da incidência, bem como da mortalidade devido ao câncer de colo do útero, não depende só da alta taxa de cobertura de citologia, mas é também preciso expertise 
adequada para a coleta do exame, resultados menos demorados e tratamento apropriado em tempo oportuno ${ }^{(15)}$.

Ainda na avaliação global da mulher que se submete ao exame colpocitológico, a queixa deve ser avaliada no momento do exame e tratada quando necessário. É importante também verificar a presença de outras doenças, como as ISTs, e realizar a prescrição de medicamentos ${ }^{(4)}$. Os profissionais não assumem o papel de prescritores de forma plena em nenhuma área de cuidado, a despeito do que rege a lei do exercício profissional da categoria. Isso se dá pelo fato de esses enfermeiros não se sentirem capacitados para tal atribuição ${ }^{(16-17)}$.

Dentre as mulheres que se encontram em situações especiais para coleta do exame citológico, situam-se as imunossuprimidas - mulheres infectadas pelo vírus HIV, ou que foram submetidas a transplante de órgãos sólidos, em tratamento de câncer e em uso crônico de corticosteroides -, que devem realizar o controle após o início da atividade sexual, no primeiro ano, com intervalos semestrais. Se os resultados forem normais, é preciso dar continuidade ao rastreamento anual. Mulheres HIV soro positivas, com CD4 inferior a 200 células $/ \mathrm{mm}^{3}$, devem corrigir os níveis e realizar o rastreamento a cada seis meses. Para o grupo de mulheres sem história de atividade sexual, não há indicação para rastrear $^{(1)}$.

No que diz respeito ao plano de cuidados na prevenção do colo do útero, as atribuições são de responsabilidade tanto de enfermeiros e médicos, quanto da equipe multiprofissional. Esse plano envolve o agendamento da consulta de retorno, o encaminhamento para serviços de referência, o acompanhamento da usuária pós-exame, o estímulo às ações de prevenção primária, as ações de vigilância em saúde e educação em saúde ${ }^{(1,4)}$.

O protocolo dá liberdade ao enfermeiro para realizar encaminhamentos (seja para ginecologia/colposcopia, seja para oncoginecologia) dos casos que necessitam de avaliação nos serviços de referência, de acordo com os critérios acordados pela gestão municipal, estadual e/ou federal, seguindo as recomendações para cada caso $^{(4)}$. Além disso, trata também da autonomia do enfermeiro diante da avaliação do resultado do citológico. No entanto, é necessário refletir se os enfermeiros, quando deixam de atender, não estão deixando também de garantir atributos da Atenção Primária à Saúde, tais como acessibilidade e integralidade da assistência.

Como visto, o resultado do citológico permite também o diagnóstico para o câncer de colo do útero. Entretanto, ele pode ocorrer tardiamente, quando está relacionado a fatores como: profissionais não capacitados na atenção oncológica, unidades de saúde inaptas para receber a demanda dessas usuárias e, ainda, a inexistência de fluxos assistenciais estabelecidos pelos gestores municipais e estaduais nos diversos pontos da rede de atenção à saúde ${ }^{(18-19)}$. Estes fluxos devem garantir a gestão do cuidado pensada de forma plena, necessitando de inúmeros serviços de saúde ${ }^{(20)}$, pois não há integralidade sem a transversalidade do sistema.

Os profissionais de enfermagem, ao realizarem atividades educativas durante a consulta, na sala de espera e nos grupos, devem enfatizar os aspectos referentes à prevenção e ao controle do câncer de colo do útero e não apenas às queixas apresentadas pelas mulheres, de modo a tornar possível intensificar as medidas preventivas $^{(21)}$.

Como forma de educação em saúde, o protocolo fala sobre a questão da orientação individual e coletiva acerca do exame e sua importância, abordando também fatores de risco, tais como tabagismo, infecção por HPV e idade. Outros temas a serem abordados na atividade educativa são o sexo seguro, a periodicidade da realização dos exames e as orientações que antecedem a coleta do material do citológico ${ }^{(4)}$.

Neste estudo, as principais facilidades acerca da implementação de ações preventivas em câncer de colo do útero, citada pelos enfermeiros, foram a busca espontânea pelo exame citopatológico e o estabelecimento de vínculo com a comunidade.

Apesar de os participantes da pesquisa terem mencionado a busca pelo citopatológico como 
uma facilidade para prevenção do câncer de colo útero, é recomendável atenção quanto ao excesso de atendimentos na demanda espontânea e a oferta irregular do exame, pois isso reflete o foco na queixa atual e deficiência no rastreamento precoce. Desse modo, ressalta-se a necessidade de conhecer a população adscrita à unidade básica de saúde, a fim de subsidiar a integralidade da assistência e a busca ativa de mulheres que se enquadram nos critérios de público-alvo para realização do citopatológico (mulheres com idade entre 25 e 64 anos e que já tiveram atividade sexual) ${ }^{(13)}$.

Na ESF, o vínculo caracteriza-se pela relação interpessoal entre o profissional de saúde e o usuário, permitindo melhor adesão aos cuidados de saúde e maior compreensão sobre as vulnerabilidades da comunidade local, por meio da escuta qualificada e do respeito mútuo. Quando se estabelece esse vínculo, o usuário deposita confiança na prática do enfermeiro, ampliando as ações de saúde e estimulando as práticas preventivas e de autocuidado ${ }^{(22)}$.

Quanto às dificuldades relatadas pelos participantes do estudo, destacam-se baixa adesão das usuárias, falta de busca ativa e escassez de treinamento para os profissionais. A baixa adesão das usuárias e a falta de busca ativa revelam problemas relacionados à organização dos fluxos de atendimento, tais como demora na marcação e no retorno dos resultados dos exames, fatores que dificultam o estabelecimento de uma intervenção precoce ${ }^{(23)}$.

Os problemas relacionados à resolutividade da rede de serviços que interferem na qualidade da atenção, como a ausência de instrumentos comunicacionais entre níveis e desorganização do processo de trabalho das equipes de saúde da família, geram desconfiança do usuário com o serviço e ainda prejudicam a coordenação do cuidado, atributo essencial da ESF, porta prioritária do sistema público de saúde ${ }^{(23)}$.

Outros obstáculos são também apontados, a exemplo de inexistência de prontuários eletrônicos nas unidades de saúde da família e nos centros de especialidades, ausência de interseção entre os diversos pontos da rede e uso limitado de protocolos clínicos. Estes empecilhos foram identificados em estudo que tratou da gestão do cuidado no Sul da Bahia e revelou que dificultavam o contato entre generalistas e especialistas, ficando a comunicação a cargo do próprio usuário $^{(24)}$.

Dificuldades foram também encontradas por enfermeiros em estudo de revisão integrativa sobre o papel desse profissional na prevenção do câncer de colo do útero, tais como excesso de trabalho, pouca disponibilidade de materiais e insumos para a realização do exame, demora na devolução dos resultados para a unidade de saúde e infraestrutura precária das Unidades de Saúde. Outras dificuldades são relacionadas ao sentimento de medo e vergonha da mulher em realizar o exame, além do preconceito dos companheiros ${ }^{(25)}$.

Por fim, no tocante à qualificação e capacitação profissional, aspecto igualmente relatado por participantes do estudo, é necessário promover ações de educação permanente, no contexto da ESF, para preencher as lacunas de conhecimento e as atitudes dos profissionais de saúde, dando subsídio para aliar teoria à prática ${ }^{(4)}$.

A limitação deste estudo refere-se ao fato de a investigação estar direcionada apenas para enfermeiros da Estratégia de Saúde da Família de um munícipio, restringindo a realidade analisada. Entretanto, estima-se que o registro sobre o uso do protocolo de saúde da mulher na prevenção do câncer de colo do útero por enfermeiros possa identificar possíveis fragilidades a respeito desse tema e nortear ações que melhorem a assistência à saúde oferecida na Atenção Básica.

\section{Conclusão}

A análise do uso do protocolo de saúde da mulher na prevenção do câncer de colo do útero por enfermeiros na Atenção Básica permitiu constatar-se que a maioria dos profissionais entrevistados fazia uso desse protocolo para subsidiar e nortear suas condutas relacionadas à prevenção e detecção precoce de câncer do colo uterino e da mama.

O olhar sobre cada dimensão do protocolo evidenciou detalhes importantes que podem implicar na melhora da qualidade da atenção à 
saúde da mulher. No Acolhimento com escuta qualificada, observou-se que o enfermeiro o realizava, limitando-se à queixa da mulher motivada por demanda espontânea.

Na Avaliação global da mulher, observou-se que a maioria dos enfermeiros realizava consulta de forma integral, não se restringindo à simples coleta do exame citológico. Em relação à intervenção em casos de alterações presentes no exame citológico, já realizavam a abordagem sindrômica - dependendo do tipo de alteração -, mas após a coleta. Outros ratificaram que só tomavam alguma atitude quando recebiam o resultado, para, possivelmente, contrastar o observado macroscópico com o microscópico e estabelecer a melhor conduta para a usuária.

No Plano de Cuidados na prevenção do câncer de colo do útero, todos os enfermeiros informaram ter autonomia para a realização do exame, no entanto nem todos realizavam avaliação do resultado desse exame. O encaminhamento das usuárias para os serviços de referência acontecia, mas não havia contrarreferência do serviço.

$\mathrm{Na}$ dimensão Facilidades e dificuldades nas ações de prevenção do câncer de colo do útero, observou-se que havia facilidades quanto à busca espontânea e o vínculo. Como dificuldades apontadas, muitas estavam relacionadas à baixa adesão das usuárias ao exame e falta de busca ativa. O estudo mostrou que os enfermeiros precisavam colaborar para a busca ativa das mulheres que nunca realizaram o exame citológico ou que há mais de três anos não o faziam, como também realizar ações educativas, a fim de mostrar-lhes que se tratava de um simples exame, porém de grande importância.

Considerando o examinado nas falas dos enfermeiros, constatou-se uma discrepância entre as ações realizadas, que ora estavam de acordo com o Protocolo de Atenção Básica, ora divergiam de suas normativas. Assim, esta pesquisa revelou fragilidade na prevenção do câncer de colo do útero, principalmente no que se refere à realização do exame citopatológico por enfermeiros na Atenção Básica.

\section{Colaborações:}

1 - concepção, projeto, análise e interpretação dos dados: Joyce Carolyne Ribeiro de Holanda, Wezila Gonçalves do Nascimento e Claudia Santos Martiniano Sousa;

2 - redação do artigo e revisão crítica relevante do conteúdo intelectual: Joyce Carolyne Ribeiro de Holanda e Maria Helloysa Herculano Pereira de Oliveira de Araújo;

3 - aprovação final da versão a ser publicada: Joyce Carolyne Ribeiro de Holanda, Maria Helloysa Herculano Pereira de Oliveira de Araújo, Wezila Gonçalves do Nascimento, Maeli Priscila Alves Gama e Claudia Santos Martiniano Sousa.

\section{Referências}

1. Brasil. Ministério da Saúde. Secretaria da Atenção à Saúde. Departamento de Atenção Básica. Controle dos cânceres do colo do útero e da mama. Cadernos de Atenção Básica n. 13 [Internet]. Brasília (DF); 2013 [cited 2020 Jul 13]. Available from: http://bvsms.saude.gov.br/bvs/ publicacoes/controle_cancer_colo_utero_mama.pdf

2. Instituto Nacional de Câncer. Estimativa 2020: incidência de câncer no Brasil [Internet]. Rio de Janeiro; 2019 [cited 2020 Jun 20]. Available from: https://www.inca.gov.br/publicacoes/livros/ estimativa-2020-incidencia-de-cancer-no-brasil

3. Barbosa DC, Lima EC. Compreensão das mulheres sobre o câncer de colo do útero e suas formas de prevenção em um município do interior da Bahia, Brasil. Rev APS [Internet]. 2016 [cited 2021 Mar 25];19(4):546-55. Available from: file://C:/Users/ Maria\%20Helloysa/Downloads/15734-Texto\%20 do\%20artigo-67512-1-10-2017 0222.pdf

4. Brasil. Ministério da Saúde, Instituto Sirio-Libanês de Ensino e Pesquisa. Protocolos da Atenção Básica: Saúde das Mulheres [Internet]. Brasília (DF): Ministério da Saúde; 2016 [cited 2020 Jun 19]. Available from: http://189.28.128.100/dab/docs/ portaldab/publicacoes/protocolo_saude_mulher. pdf

5. Melo MCSC, Vilela F, Salimena AMO, Souza IEO. O Enfermeiro na Prevenção do Câncer do Colo do Útero: o Cotidiano da Atenção Primária. Rev Bras Cancerol [Internet]. 2012 [cited 2017 Jan 
26];58(3):389-98. Available from: https://rbc. inca.gov.br/revista/index.php/revista/article/ view/590/364

6. Rosso CFW, Cruvinel KPDS, Silva MADS, Almeida NAM, Pereira VM, Pinheiro DCDS organizadores. Protocolo de enfermagem na atenção primária à saúde no estado de Goiás [Internet]. 2a ed. Goiânia: Conselho Regional de Enfermagem de Goiás; 2014 [cited 2020 May 15]. Available from: http://www.corengo.org. br/wp-content/uploads/2015/02/Protocolo-deEnfermagem-2015.pdf

7. Bardin L. Análise de conteúdo. Lisboa (PT): Edições 70; 2016.

8. Lima MADDS, Ramos DD, Rosa RB, Nauderer TM, Davis R. Acesso e acolhimento em unidades de saúde na visão dos usuários. Acta paul enferm. 2007;20(1):12-7. DOI: 10.1590/ S0103-21002007000100003

9. Ferreira MLSM. Motivos que influenciam a não-realização do exame de papanicolaou segundo a percepção de mulheres. Esc Anna Nery. 2009;13(2):378-84. DOI: 10.1590/ S1414-81452009000200020

10. Dantas CN, Enders BC, Salvado PTCO. Experiência da enfermeira na prevenção do câncer cérvico-uterino. Rev Baiana Saúde Pública. 2011;35(3):646-60. DOI: 10.22278/2318-2660.2011. v35.n3.a284

11. Fuerwerker LCM. Cuidar em saúde. In: Feuerwerker LCM, Bertussi DC, Merhy EE, organizadores. Avaliação compartilhada do cuidado em saúde: surpreendendo o instituído nas redes [Internet]. Rio de Janeiro: Hexis; 2016. p. 35-47 [cited 2017 Jan 28]. Available from: http://historico. redeunida.org.br/editora/biblioteca-digital/ colecao-micropolitica-do-trabalho-e-o-cuidadoem-saude/politicas-e-cuidados-em-saude-livro-2avaliacao-compartilhada-do-cuidado-em-saudesurpreendendo-o-instituido-nas-redes-pdf

12. Paiva CCN, Caetano R. Evaluation of the implementation of sexual and reproductive health actions in Primary Care: scope review. Esc Anna Nery. 2020;24(1):e20190142. DOI: https://doi.org/10.1590/2177-9465-ean-2019-0142

13. Nasser MA, Nemes MIB, Andrade MC, Prado RR, Castanheira ERL. Avaliação na atenção primária paulista: ações incipientes em saúde sexual e reprodutiva. Rev Saúde Pública.
2017;51:77. DOI: https://doi.org/10.11606/s15188787.2017051006711

14. Barbosa TLA, Gomes LMX, Holzmann APF, Paula AMB, Haika DSA. Counseling about sexually transmitted diseases in primary care: perception and professional practice. Acta Paul Enferm. 2015;28(6):531-8. DOI: https://doi. org/10.1590/1982-0194201500089

15. Quadros CATD, Victora CG, Costa JSDD. Coverage and focus of a cervical cancer prevention program in southern Brazil. Rev Panam Salud Publica. 2004 Oct;16(4):223-32. DOI: $10.1590 /$ s1020-49892004001000001

16. Martiniano CS, Coêlho AA, Latter S, Uchoa SAC. Medication prescription by nurses and the case of the Brazil: what can we learn from international research? Int J Nurs Stud. 2014 Aug;51(8):1071-3. DOI: 10.1016/j.ijnurstu.2013. 12.006

17. Martiniano CS, Andrade PS, Magalhães FC, Souza FF, Clementino FS, Uchôa SAC. Legalização da prescrição de medicamentos pelo enfermeiro no Brasil: história, tendências e desafios. Texto Contexto Enferm. 2015;24(3):809-17. DOI: https://doi.org/10.1590/0104-070720150017 20014

18. Mendonça VGD, Lorenzato FRB, Mendonça JG, Menezes TC, Guimarães MJB. Mortalidade por câncer do colo do útero: características sociodemográficas das mulheres residentes na cidade de Recife, Pernambuco. Rev Bras Ginecol Obstet. 2008;30(5):248-55. DOI: http:// dx.doi.org/10.1590/S0100-72032008000500007

19. Instituto Nacional de Câncer, Ministério da Saúde. Nomenclatura Brasileira para Laudos Cervicais e Condutas Preconizadas: recomendações para profissionais de saúde. J Bras Patol Med Lab [Internet]. 2006 [cited 2017 Jan 8];42(5):351-73. Available from: http://www.scielo.br/pdf/jbpml/ v42n5/a08v42n5.pdf

20. Cecilio LCO. A morte de Ivan llitch, de Leon Tolstói: elementos para se pensar as múltiplas dimensões da gestão do cuidado. Interface (Botucatu). 2009;13(supl.1):545-55. DOI: https:// doi.org/10.1590/S1414-32832009000500007

21. Guimarães JAF, Aquino PS, Pinheiro AKB, Moura JG. Pesquisa brasileira sobre prevenção do câncer de colo uterino: uma revisão integrativa. Rev Rene [Internet]. 2012 [cited 2017 
Nov 3];13(1):220-30. Available from: http://www. periodicos.ufc.br/rene/article/view/3797

22. Brunello MEF, Ponce MAZ, Assis EG, Andrade RLP, Scatena LM, Palha PF, et al. O vínculo na atenção à saúde: revisão sistematizada na literatura, Brasil (1998-2007). Acta Paul Enferm. 2010; 23(1):131-5. DOI: https:// doi.org/10.1590/S0103-21002010000100021

23. Almeida PF, Gérvas J, Freire J-M, Giovanella L. Estratégias de integração entre atenção primária à saúde e atenção especializada: paralelos entre Brasil e Espanha. Saúde Debate. 2013;37(98):400-15. DOI: https://doi.org/10.1590/ S0103-11042013000300004
24. Santos AM, Giovanella L. Gestão do cuidado integral: estudo de caso em região de saúde da Bahia, Brasil. Cad Saúde Pública. 2016;32(3):e00172214. DOI: 10.1590/0102-311X00 172214

25. Paiva ARO, Nunes PBS, Vale GMVF, Prudêncio FA, Silva RF, Nôleto JS, et al. O enfermeiro da atenção básica na prevenção do câncer do colo do útero: revisão integrativa. Rev UNINGÁ [Internet]. 2017 [cited 2017 nov 9];52(1):162-5. Available from: http://34.233.57.254/ index.php/uninga/article/view/1372

Recebido: 28 de setembro de 2020

Aprovado: 25 de março de 2021

Publicado: 29 de abril de 2021

A Revista Baiana de Enfermagem utiliza a Licença Creative Commons - Atribuição-NãoComercial 4.0 Internacional.

https://creativecommons.org/licenses/by-nc/4.0/

Este artigo é de acesso aberto distribuído sob os termos da Licença Creative Commons (CC BY-NC).

Esta licença permite que outros remixem, adaptem e criem a partir do seu trabalho para fins não comerciais. Embora os novos trabalhos tenham de lhe atribuir o devido crédito e não possam ser usados para fins comerciais, os usuários não têm de licenciar esses trabalhos derivados sob os mesmos termos. 